\title{
Separation of variables and explicit theta-function solution of the classical Steklov-Lyapunov systems: A geometric and algebraic geometric background.
}

\author{
Yuri Fedorov \\ e-mail: Yuri.Fedorov@upc.edu \\ Department de Matemàtica Aplicada I, \\ Universitat Politecnica de Catalunya, \\ Barcelona, E-08028 Spain \\ and \\ Inna Basak \\ Department de Matemàtica Aplicada I, \\ Universitat Politecnica de Catalunya, \\ Barcelona, E-08028 Spain \\ e-mail: Inna.Basak@upc.edu
}

\begin{abstract}
The paper revises the separation of variables and explicit integration of the classical SteklovLyapunov systems, which was first made by F. Kötter in 1900. Namely, we give a geometric interpretation of the separating variables and, then, applying the Weierstrass root functions, obtain an explicit theta-function solution to the problem. We also give such a solution for an alternative set of phase variables of the systems that has a much simpler form.
\end{abstract}

\section{Introduction}

The motion of a rigid body in the ideal incompressible fluid is described by the classical Kirchhoff equations

$$
\dot{K}=K \times \frac{\partial H}{\partial K}+p \times \frac{\partial H}{\partial p}, \quad \dot{p}=p \times \frac{\partial H}{\partial p},
$$

where $K, p \in \mathbb{R}^{3}$ are the vectors of the impulsive momentum and the impulsive force, and $H=$ $H(K, p)$ is the Hamiltonian, which is quadratic in $K, p$. Note that this system always possesses two trivial integrals (Casimir functions of the coalgebra $\left.e^{*}(3)\right)\langle K, p\rangle,\langle p, p\rangle$ and the Hamiltonian itself is also a first integral.

Steklov [15] noticed that the classical Kirchhoff equations are integrable under certain conditions i.e., when the Hamiltonian has the form

$$
H_{1}=\frac{1}{2} \sum_{\alpha=1}^{3}\left(b_{\alpha} K_{\alpha}^{2}+2 \nu b_{\beta} b_{\gamma} K_{\alpha} p_{\alpha}+\nu^{2} b_{\alpha}\left(b_{\beta}-b_{\gamma}\right)^{2} p_{\alpha}^{2}\right), \quad(\alpha, \beta, \gamma)=(1,2,3),
$$

$b_{1}, b_{2}, b_{3}$ and $\nu$ being arbitrary parameters. Under the Steklov condition, the equations possess fourth additional integral

$$
H_{2}=\frac{1}{2} \sum_{\alpha=1}^{3}\left(K_{\alpha}^{2}-2 \nu b_{\alpha} K_{\alpha} p_{\alpha}+\nu^{2}\left(b_{\beta}-b_{\gamma}\right)^{2} p_{\alpha}^{2}\right) .
$$

\footnotetext{
${ }^{*}$ AMS Subject Classification 37J60, 37J35, 70H45
} 
Later Lyapunov [16] discovered an integrable case of the Kirchhoff equations whose Hamiltonian was a linear combination of the additional integral (2) and the two trivial integrals. Thus, the Steklov and Lyapunov integrable systems actually define different trajectories on the same invariant manifolds, two-dimensional tori. This fact was first noticed in [12].

In the sequel, without loss of generality, we assume $\nu=1$ (this can always be made by an appropriate rescaling $p \rightarrow p / \nu)$.

The Kirchhoff equations with the Hamiltonians (1), (2) were first solved explicitly by Kötter [14], who used the change of variables $(K, p) \rightarrow(z, p)$ :

$$
2 z_{\alpha}=K_{\alpha}-\left(b_{\beta}+b_{\gamma}\right) p_{\alpha}, \quad \alpha=1,2,3, \quad(\alpha, \beta, \gamma)=(1,2,3)
$$

which transforms the Steklov-Lyapunov systems to the form

$$
\dot{z}=z \times B z-B p \times B z, \quad \dot{p}=p \times B z, \quad B=\operatorname{diag}\left(b_{1}, b_{2}, b_{3}\right)
$$

and, respectively,

$$
\dot{z}=p \times B z, \quad \dot{p}=p \times(z-B p) .
$$

Kötter implicitly showed that the above systems admit the following Lax representation with $3 \times 3$ skew-symmetric matrices and a spectral parameter

$$
\begin{aligned}
\dot{L}(s) & =[L(s), A(s)], \quad L(s), A(s) \in s o(3), \quad s \in \mathbb{C}, \\
L(s)_{\alpha \beta} & =\varepsilon_{\alpha \beta \gamma}\left(\sqrt{s-b_{\gamma}}\left(z_{\gamma}+s p_{\gamma}\right)\right),
\end{aligned}
$$

where $\varepsilon_{\alpha \beta \gamma}$ is the Levi-Civita tensor. Equations (4) and (5) are generated by the operators

$$
A(s)_{\alpha \beta}=\frac{\varepsilon_{\alpha \beta \gamma}}{s} \sqrt{\left(s-b_{\alpha}\right)\left(s-b_{\beta}\right)} b_{\gamma} z_{\gamma}, \quad \text { resp. } \quad A(s)_{\alpha \beta}=\varepsilon_{\alpha \beta \gamma} \sqrt{\left(s-b_{\alpha}\right)\left(s-b_{\beta}\right)} p_{\gamma} .
$$

The radicals in (6)-(7) are single-valued functions on the elliptic curve $\widehat{\mathcal{E}}$, the 4 -sheeted unramified covering of the plane curve $\mathcal{E}=\left\{w^{2}=\left(s-b_{1}\right)\left(s-b_{2}\right)\left(s-b_{3}\right)\right\}$. For this reason, the Lax representation has an elliptic spectral parameter.

Writing out the characteristic equation for $L(s)$, we arrive at the following family of quadratic integrals

$$
\mathcal{F}(s)=\sum_{\gamma=1}^{3}\left(s-b_{\gamma}\right)\left(z_{\gamma}+s p_{\gamma}\right)^{2} \equiv J_{1} s^{3}+J_{2} s^{2}+2 s H_{2}-2 H_{1}
$$

where

$$
H_{1}=\frac{1}{2}\langle z, B z\rangle, \quad H_{2}=\frac{1}{2}\langle z, z\rangle-\langle B z, p\rangle, \quad J_{2}=2\langle z, p\rangle-\langle B p, p\rangle, \quad J_{1}=\langle p, p\rangle .
$$

It is seen that under the Kötter substitution (3) the functions $J_{1}, J_{2}$ transform into invariants of the coalgebra $e^{*}(3)$, whereas the integrals $H_{1}(z, p), H_{2}(z, p)$ (up to a linear combination of the invariants) become the Hamiltonians (1),(2).

An analog of the Lax pair (6) was later rediscovered in [5] and was used to obtain theta-function solution of the systems by using the method of Baker-Akhieser functions (see [4]). However, the resulting formulas appeared to be quite tedious, and it was not evident how to compare or identify them with the theta-function solution of Kötter.

Note that the latter was obtained in the classical manner, i.e., by a separation of variables and reduction of the equations of motion to quadratures, which have the form of the Abel-Jacobi map associated to a genus 2 hyperelliptic curve. The phase variables of the Kirchhoff equations have been expressed in terms of the separating variables in a quite symmetric but complicated way. Until recently, various attempts to check these expressions, as well as the reduction to quadratures made by Kötter, even using packages of modern computer algebra, were not successful. This even led to an opinion among some specialists that the results of [14] are not reliable hence useless. 
One of the first step in verification of Kötters' calculations was made in [7], where the SteklovLyapunov systems on $e^{*}(3)$, as well as their higher-dimensional generalizations, have been considered as Poisson reductions of certain Hamiltonian systems in a bigger phase space. The latter systems were shown to possess $2 \times 2$ matrix Lax representations in a generalized Gaudin form with a rational spectral parameter. This fact easily allowed to find separating variables, which coincided with those suggested by Kötter, and, as a byproduct, prove their commutativity with respect to the Lie-Poisson bracket on $e^{*}(3)$. A similar approach to the separation of variables was made in [18].

The main aim of the present paper is to reconstruct the rest of the results of the paper $[14]^{1}$.

For our purposes we shall also use another set of phase variables which depend linearly on $z, p$. Namely, putting in (8) successively $s=b_{1}, s=b_{2}, s=b_{3}$ we obtain three independent quadratic integrals defining rank 3 quadrics in $\mathbb{P}^{6}$ :

$$
\begin{aligned}
& \left(b_{1}-b_{2}\right)\left(z_{2}+b_{1} p_{2}\right)^{2}+\left(b_{1}-b_{3}\right)\left(z_{3}+b_{1} p_{3}\right)^{2}=\mathcal{F}\left(b_{1}\right), \\
& \left(b_{2}-b_{1}\right)\left(z_{1}+b_{2} p_{1}\right)^{2}+\left(b_{2}-b_{3}\right)\left(z_{3}+b_{2} p_{3}\right)^{2}=\mathcal{F}\left(b_{2}\right), \\
& \left(b_{3}-b_{1}\right)\left(z_{1}+b_{3} p_{1}\right)^{2}+\left(b_{3}-b_{2}\right)\left(z_{2}+b_{3} p_{2}\right)^{2}=\mathcal{F}\left(b_{3}\right) .
\end{aligned}
$$

Then it is natural to introduce new variables

$$
\begin{aligned}
& v_{1}=\sqrt{\left(b_{2}-b_{3}\right)\left(b_{1}-b_{2}\right)}\left(z_{2}+b_{1} p_{2}\right), \\
& v_{2}=\sqrt{\left(b_{2}-b_{3}\right)\left(b_{3}-b_{1}\right)}\left(z_{3}+b_{1} p_{3}\right), \\
& v_{3}=\sqrt{\left(b_{3}-b_{1}\right)\left(b_{1}-b_{2}\right)}\left(z_{1}+b_{2} p_{1}\right), \\
& v_{4}=\sqrt{\left(b_{2}-b_{3}\right)\left(b_{3}-b_{1}\right)}\left(z_{3}+b_{2} p_{3}\right), \\
& v_{5}=\sqrt{\left(b_{3}-b_{1}\right)\left(b_{1}-b_{2}\right)}\left(z_{1}+b_{3} p_{1}\right), \\
& v_{6}=\sqrt{\left(b_{2}-b_{3}\right)\left(b_{1}-b_{2}\right)}\left(z_{2}+b_{3} p_{2}\right),
\end{aligned}
$$

which, in particular, imply

$$
\begin{gathered}
p_{1}=\frac{v_{3}-v_{5}}{\sqrt{\mathcal{S} \sqrt{b_{2}-b_{3}}}}, \quad p_{2}=\frac{v_{1}-v_{6}}{\sqrt{\mathcal{S} \sqrt{b_{3}-b_{1}}}}, \quad p_{3}=\frac{v_{2}-v_{4}}{\sqrt{\mathcal{S} \sqrt{b_{1}-b_{2}}}}, \\
\mathcal{S}=\left(b_{1}-b_{2}\right)\left(b_{2}-b_{3}\right)\left(b_{3}-b_{1}\right) .
\end{gathered}
$$

Then the integrals $(10)$ and $(p, p)=J_{1}$ take the following compact form

$$
\begin{gathered}
v_{1}^{2}-v_{2}^{2}=\psi\left(b_{1}\right) /\left(b_{2}-b_{3}\right), \\
v_{3}^{2}-v_{4}^{2}=\psi\left(b_{2}\right) /\left(b_{3}-b_{1}\right), \\
v_{5}^{2}-v_{6}^{2}=\psi\left(b_{3}\right) /\left(b_{1}-b_{2}\right), \\
\frac{\left(v_{3}-v_{5}\right)^{2}}{b_{2}-b_{3}}+\frac{\left(v_{1}-v_{6}\right)^{2}}{b_{3}-b_{1}}+\frac{\left(v_{2}-v_{4}\right)^{2}}{b_{1}-b_{2}}=J_{1}\left(b_{1}-b_{2}\right)\left(b_{2}-b_{3}\right)\left(b_{3}-b_{1}\right) .
\end{gathered}
$$

The Steklov-Lyapunov systems written in terms of $v_{1}, \ldots, v_{6}$, as well as the integrals (12), are quite similar to those describing the reduction of the integrable geodesic flow on the group $S O(4)$ with the diagonal metric II to the algebra so(4), which was considered in details in $[1,2]$. In fact, as was shown by several authors (see e.g., [5]), there is a linear isomorphism connecting the above systems. We shall use this property and the results of [2] to obtain theta function expressions for the sums and differences of $v_{i}$, which have an especially simple form.

\section{Separation of variables by F. Kötter.}

The explicit solution of the Steklov-Lyapunov systems in the generic case was given by Kötter in the brief communication [14], where he presented the following scheme.

\footnotetext{
${ }^{1}$ Note that apart from the solutions of the Kirchhoff equations, Kötter also provided (although in an extremely brief form) the theta-solutions describing the motion of the group $E(3)$, that is, the components of the rotation matrix of the body and the trajectory of its center in space. We could not reconstruct these solutions.
} 
Let us fix the constants of motion, then the invariant polynomial (8) can be written as

$$
\mathcal{F}(s)=c_{0}\left(s-c_{1}\right)\left(s-c_{2}\right)\left(s-c_{3}\right), \quad c_{0}, c_{1}, \ldots, c_{3}=\text { const. }
$$

Assume, without loss of generality, that $b_{1}<b_{2}<b_{3}$. Then one can show that for real $z, p$ there are two possibilities:

1) $c_{1}, c_{2}, c_{3}$ are all real, then $b_{1} \leq c_{1} \leq c_{2} \leq c_{3} \leq b_{3}$;

2) $c_{1}$ is real and $c_{2}, c_{3}$ are complex conjugated, then $b_{1} \leq c_{1} \leq b_{3}$ and either $\rho=\Re c_{2}=\Re c_{3}<b_{1}$ or $\rho>b_{3}$.

Next, when no one of $c_{\alpha}$ coincides with $b_{1}, b_{2}, b_{3}$, the level variety of the four first integrals of the problem (given by the coefficients at $s^{3}, s^{2}, s, s^{0}$ ) is a union of two-dimensional tori in $\mathbb{R}^{6}=(z, p)$. We restrict ourselves to this generic situation, excluding the other cases, which correspond to special motions.

Let $\lambda_{1}, \lambda_{2}$ be the roots of the equation

$$
f(\lambda)=\sum_{i=1}^{3} \frac{\left(z_{j} p_{k}-z_{k} p_{j}\right)^{2}}{\lambda-b_{i}}=0, \quad(i, j, k)=(1,2,3)
$$

where, when all $c_{\alpha}$ are real,

$$
\lambda_{1} \in\left[b_{1}, c_{1}\right], \quad \lambda_{2} \in\left[c_{3}, b_{3}\right] .
$$

Then for fixed $c_{0}, c_{1}, c_{2}, c_{3}$ the variables $z, p$ can be expressed in terms of $\lambda_{1}, \lambda_{2}$ in such a way that for any $s \in \mathbb{C}$ the following relation holds (see formula (7) in [14])

$$
z_{i}+s p_{i}=\sqrt{c_{0}} \frac{x_{i} \sum_{\alpha=1}^{3}\left(s-c_{\alpha}\right) \frac{\sqrt{-\left(\lambda_{1}-c_{\alpha}\right)\left(\lambda_{2}-c_{\alpha}\right)}}{\left(c_{\alpha}-c_{\beta}\right)\left(c_{\alpha}-c_{\gamma}\right)}\left(\frac{\sqrt{\Phi\left(\lambda_{1}\right) \psi\left(\lambda_{2}\right)}}{\left(\lambda_{1}-b_{i}\right)\left(\lambda_{2}-c_{\alpha}\right)}-\frac{\sqrt{\Phi\left(\lambda_{2}\right) \psi\left(\lambda_{1}\right)}}{\left(\lambda_{2}-b_{i}\right)\left(\lambda_{1}-c_{\alpha}\right)}\right)}{\left(\lambda_{1}-\lambda_{2}\right) \sum_{\alpha=1}^{3} \frac{\sqrt{-\left(\lambda_{1}-c_{\alpha}\right)\left(\lambda_{2}-c_{\alpha}\right)}}{\left(c_{\alpha}-c_{\beta}\right)\left(c_{\alpha}-c_{\gamma}\right)}}
$$

where

$$
\begin{gathered}
\Phi(\lambda)=\left(\lambda-b_{1}\right)\left(\lambda-b_{2}\right)\left(\lambda-b_{3}\right), \quad \psi(\lambda)=\left(\lambda-c_{1}\right)\left(\lambda-c_{2}\right)\left(\lambda-c_{3}\right), \\
x_{i}=\frac{\sqrt{\left(\lambda_{1}-b_{i}\right)\left(\lambda_{2}-b_{i}\right)}}{\sqrt{\left(b_{i}-b_{j}\right)\left(b_{i}-b_{k}\right)}} \\
(i, j, k)=(1,2,3), \quad(\alpha, \beta, \gamma)=(1,2,3) .
\end{gathered}
$$

Setting in the above expression $s \rightarrow \infty$ and $s=0$, one obtains the corresponding formulas for $p_{i}, z_{i}$.

Note that for real $z_{i}, p_{i}$, in the case (1) (all $c_{\alpha}$ are real), in view of the condition (15) all the expressions under the radicals in (16) are non-negative. In the rest of the cases the roots can be complex. For any $\alpha=1,2,3$, the branches of $\sqrt{-\left(\lambda_{1}-c_{\alpha}\right)\left(\lambda_{2}-c_{\alpha}\right)}$ in the numerator and the denominator of (16) must be the same.

Next, the evolution of $\lambda_{1}, \lambda_{2}$ is described by the quadratures

$$
\begin{gathered}
\frac{d \lambda_{1}}{\sqrt{R\left(\lambda_{1}\right)}}+\frac{d \lambda_{2}}{\sqrt{R\left(\lambda_{2}\right)}}=\delta_{1} d t \\
\frac{\lambda_{1} d \lambda_{1}}{\sqrt{R\left(\lambda_{1}\right)}}+\frac{\lambda_{2} d \lambda_{2}}{\sqrt{R\left(\lambda_{2}\right)}}=\delta_{2} d t \\
R(\lambda)=-\Phi(\lambda) \psi(\lambda)
\end{gathered}
$$

with certain constants $\delta_{1}, \delta_{2}$ depending on the choice of the Hamiltonian only. Note that the paper [14] does not describe explicitly this dependence, which can be found in [7], [18]. 
The above quadratures rewritten in the integral form

$$
\begin{gathered}
\int_{\lambda_{0}}^{\lambda_{1}} \frac{d \lambda}{2 \sqrt{R(\lambda)}}+\int_{\lambda_{0}}^{\lambda_{2}} \frac{d \lambda}{2 \sqrt{R(\lambda)}}=u_{1}, \\
\int_{\lambda_{0}}^{\lambda_{1}} \frac{\lambda d \lambda}{2 \sqrt{R(\lambda)}}+\int_{\lambda_{0}}^{\lambda_{1}} \frac{\lambda d \lambda}{2 \sqrt{R(\lambda)}}=u_{2}, \\
u_{1}=\delta_{1} t+u_{10}, \quad u_{2}=\delta_{2} t+u_{20},
\end{gathered}
$$

which represent the Abel-Jacobi map associated to the genus 2 hyperelliptic curve $\mu^{2}=-\Phi(\lambda) \psi(\lambda)$. Inverting the map (20) and substituting symmetric functions of $\lambda_{1}, \lambda_{2}, \mu_{1}, \mu_{2}$ into (16), one finally finds $z, p$ as functions of time.

Everyone who had read paper [14] might be surprised by how Kötter managed to invent the intricate substitution $(z, p) \rightarrow\left(\lambda_{1}, \lambda_{2}, c_{0}, c_{1}, c_{2}, c_{3}\right)$ and to represent the result in the symmetric form (16). Unfortunately, the author of the paper gave no explanations of his computations. Nevertheless, it is clear that behind the striking formulas there must be a certain geometric idea, which we try to reconstruct in the next section.

\section{A geometric background of Kötter's solution.}

Let $\left(x_{1}: x_{2}: x_{3}\right)$ be homogeneous coordinates in $\mathbb{P}^{2}$ defined up to multiplication by the same non-zero factor. Consider a line $l$ in $\mathbb{P}^{2}=\left(x_{1}: x_{2}: x_{3}\right)$ defined by equation

$$
y_{1} x_{1}+y_{2} x_{2}+y_{3} x_{3}=0
$$

Following Plücker (see e.g., [11]), the coefficients $y_{1}, y_{2}, y_{3}$ can be regarded as homogeneous coordinates of a point in the dual projective space $\left(\mathbb{P}^{2}\right)^{*}$. Now let $l_{1}, l_{2}$ be two intersecting lines in $\mathbb{P}^{2}$ with the Plücker coordinates $\left(y_{1}^{(1)}, y_{2}^{(1)}, y_{3}^{(1)}\right),\left(y_{1}^{(2)}, y_{2}^{(2)}, y_{3}^{(2)}\right)$.

Then, for any constants $\lambda, \mu \in \mathbb{C}$ not vanishing simultaneously, the linear combination $\lambda y_{\alpha}^{(1)}+$ $\mu y_{\alpha}^{(2)}$ are also Plücker coordinates of a line $l_{\lambda, \mu} \in \mathbb{P}^{2}$. Hence, we arrive at an important geometric object, a pencil of lines in $\mathbb{P}^{2}$, i.e., a one-parameter family $l_{\lambda, \mu}$. It is remarkable that all the lines of a pencil intersect at the same point $\mathcal{P} \in \mathbb{P}^{2}$. The point $\mathcal{P}$ is called the focus of the pencil.

Theorem 1. ([11]) Let $l_{\lambda, \mu}$ be a pencil of lines in $\mathbb{P}^{2}$ defined by Plücker coordinates $\lambda y_{\alpha}^{(1)}+\mu y_{\alpha}^{(2)},(\lambda$ : $\mu) \in \mathbb{P}$. Then the homogeneous coordinates of the focus are

$$
\mathbf{P}=\left(y_{2}^{(1)} y_{3}^{(2)}-y_{3}^{(1)} y_{2}^{(2)}: y_{1}^{(1)} y_{3}^{(2)}-y_{3}^{(1)} y_{1}^{(2)}: y_{1}^{(1)} y_{2}^{(2)}-y_{2}^{(1)} y_{1}^{(2)}\right)
$$

Next, consider the family of confocal quadrics in $\mathbb{P}^{2}$

$$
Q(s)=\left\{\frac{x_{1}^{2}}{s-b_{1}}+\frac{x_{2}^{2}}{s-b_{2}}+\frac{x_{3}^{2}}{s-b_{3}}=0\right\}
$$

and a fixed point $P=\left(X_{1}: X_{2}: X_{3}\right)$. Then one defines the spheroconical coordinates $\lambda_{1}, \lambda_{2}$ of this point (with respect to $Q(s)$ ) as the roots of the equation

$$
\frac{X_{1}^{2}}{\lambda-b_{1}}+\frac{X_{2}^{2}}{\lambda-b_{2}}+\frac{X_{3}^{2}}{\lambda-b_{3}}=0 .
$$

Now, going back to the Steklov-Lyapunov systems, we make the following observation.

Proposition 2. The separating variables $\lambda_{1}, \lambda_{2}$ defined by formula (14) are spheroconical coordinates of the focus $\mathbf{P}$ of the pencil of lines in $\mathbb{P}^{2}$ with the Plücker coordinates $z+s p=\left(z_{1}+s p_{1}: z_{2}+s p_{2}\right.$ : $\left.z_{3}+s p_{3}\right), s \in \mathbb{P}$ with respect to the family of quadrics (22). 
Proof. According to Theorem 1, the homogeneous coordinates of the focus $\mathbf{P}$ are

$$
\left(z_{2} p_{3}-z_{3} p_{2}: z_{3} p_{1}-z_{1} p_{3}: z_{1} p_{2}-z_{2} p_{1}\right)
$$

hence, the spheroconical coordinates of $\mathbf{P}$ with respect to the family (22) are precisely the roots of the equation (14), i.e., $\lambda_{1}, \lambda_{2}$.

Note also the following property: for $\alpha=1,2,3$, the line $\ell_{\alpha}$ with the Plücker coordinates $z+c_{\alpha} p$ is tangent to the quadric $Q_{\alpha}=Q\left(c_{\alpha}\right)$. Indeed, setting in the right hand side of (8) $s=c_{\alpha}$, we obtain

$$
\sum_{i=1}^{3}\left(c_{\alpha}-b_{i}\right)\left(z_{i}+c_{\alpha} p_{i}\right)^{2}=0,
$$

which represents the condition of tangency of the line $\ell_{\alpha}$ and the quadric $Q_{\alpha}$.

As a result, the following configuration holds: the three lines $\ell_{1}, \ell_{2}, \ell_{3}$ in $\mathbb{P}^{2}$ intersect at the same point $\mathbf{P}$ and are tangent to the quadrics $Q_{1}, Q_{2}, Q_{3}$ respectively. An example of such a configuration is shown in Fig. 1.

It follows that a solution $z(t), p(t)$ defines a trajectory of the focus $\mathbf{P}$ on $\mathbb{P}^{2}$ or on $S^{2}=\left\{x_{1}^{2}+x_{2}^{2}+\right.$ $\left.x_{3}^{2}=1\right\}$, and it natural to suppose that the Steklov-Lyapunov systems define dynamical systems on the sphere. Indeed, some of these systems were studied in [18] and were shown to be related to a generalization of the Neumann system with a quartic potential.

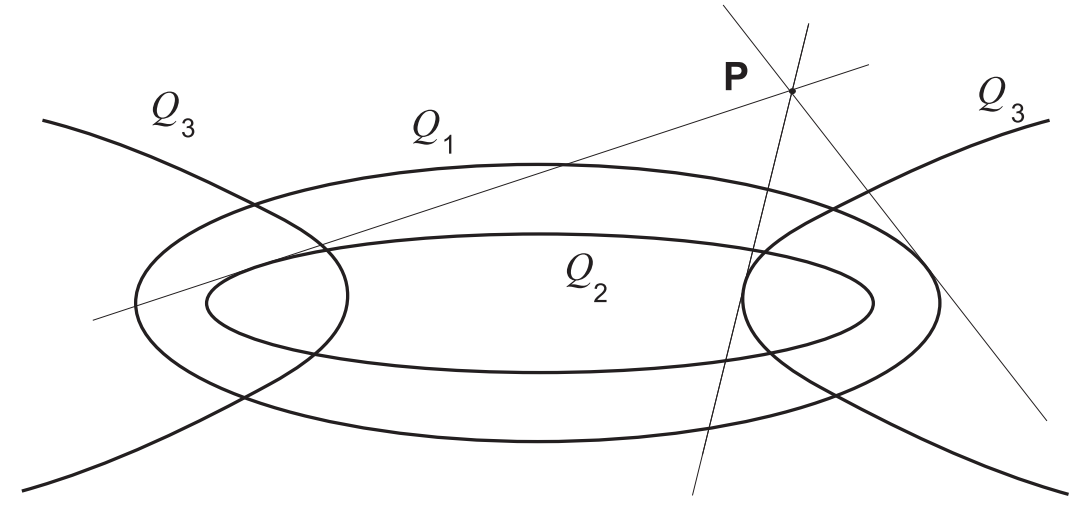

Figure 1: A configuration of tangent lines in $\mathbb{R}^{2}=\left(\frac{x_{1}}{x_{3}}, \frac{x_{2}}{x_{3}}\right)$ for the case $b_{1}<c_{1}<b_{2}<c_{2}<c_{3}<b_{3}$, when the quadrics $Q_{\alpha}$ are two ellipses and a hyperbola.

In the sequel our main goal will be to recover the variables $z$ and $p$ as functions of the spheroconical coordinates of the focus $\mathbf{P}$, that is, to reconstruct the Kötter formula (16). Obviously, the solution is not unique: to each pair $\left(\lambda_{1}, \lambda_{2}\right), \lambda_{k} \neq b_{1}, b_{2}, b_{3}$ there correspond 4 points on $\mathbb{P}^{2}$, and for each point $\mathbf{P}$ that does not lie on any of the quadrics $Q\left(c_{\alpha}\right), 2^{3}=8$ different configurations of tangent lines $\ell_{1}, \ell_{2}, \ell_{3}$ are possible (Fig. 1 shows just one of them). Thus, under the above generality conditions, a pair $\left(\lambda_{1}, \lambda_{2}\right)$ gives 32 different tangent configurations.

Reconstruction of $z, p$ in terms of the separating variables. Let $\left(\mathbb{P}^{2}\right)^{*}=\left(G_{1}: G_{2}: G_{3}\right)$ be the dual space to $\mathbb{P}^{2}=\left(x_{1}: x_{2}: x_{3}\right),\left(G_{i}\right.$ being the Plücker coordinates of lines in $\left.\mathbb{P}^{2}\right)$. It is convenient to regard $G_{i}$ also as Cartesian coordinates in the space $\left(\mathbb{C}^{3}\right)^{*}=\left(G_{1}, G_{2}, G_{3}\right)$. The pencil $\sigma(\mathbf{P})$ of lines in $\mathbb{P}^{2}$ with the focus (23) is represented by a line in $\left(\mathbb{P}^{2}\right)^{*}$ or by plane

$$
\pi=\left\{\left(z_{2} p_{3}-z_{3} p_{2}\right) G_{1}+\left(z_{3} p_{1}-z_{1} p_{3}\right) G_{2}+\left(z_{1} p_{2}-z_{2} p_{1}\right) G_{3}=0\right\} \subset\left(\mathbb{C}^{3}\right)^{*} .
$$

Consider the line $\bar{\sigma}(\mathbf{P})=\{z+s p \mid s \in \mathbb{R}\} \subset\left(\mathbb{C}^{3}\right)^{*}$. Obviously, $\{z+s p\} \subset \pi$. Now let us use the condition for the three lines $\ell_{1}, \ell_{2}, \ell_{3}$ defined by the points $z+c_{1} p, z+c_{2} p, z+c_{3} p$ in $\left(\mathbb{P}^{2}\right)^{*}$ to be 
tangent to the quadrics $Q\left(c_{1}\right), Q\left(c_{2}\right), Q\left(c_{3}\right)$ respectively. Let $\mathbf{V}_{\alpha}=\left(V_{\alpha 1}, V_{\alpha 2}, V_{\alpha 3}\right) \subset \pi, \alpha=1,2,3$ be some vectors in $\left(\mathbb{C}^{3}\right)^{*}$ representing these points, so that $\ell_{\alpha}=\left\{V_{\alpha 1} x_{1}+V_{\alpha 2} x_{2}+V_{\alpha 3} x_{3}=0\right\}$. Then we have

$$
z+c_{1} p-\mu_{1} \mathbf{V}_{1}=0, \quad z+c_{2} p-\mu_{2} \mathbf{V}_{2}=0, \quad z+c_{3} p-\mu_{3} \mathbf{V}_{3}=0
$$

for some indefinite factors $\mu_{\alpha}$. This system is equivalent to a homogeneous system of 9 scalar equations for 9 variables $z_{\alpha}, p_{\alpha}, \mu_{\alpha}, \alpha=1,2,3$. Thus the variables can be found up to multiplication by a common factor. Eliminating $z, p$ from (24), we obtain the following homogeneous system for $\mu_{1}, \mu_{2}, \mu_{3}$

$$
\left(c_{2}-c_{3}\right) V_{\alpha 1} \mu_{1}+\left(c_{3}-c_{1}\right) V_{\alpha 2} \mu_{2}+\left(c_{1}-c_{2}\right) V_{\alpha 3} \mu_{3}=0, \quad \alpha=1,2,3,
$$

which has a nontrivial solution, since $\operatorname{det}\left\|V_{\alpha i}\right\|=0$ (the vectors $\mathbf{V}_{\alpha}$ lie in the same hyperplane $\pi$ ). It follows, for example, that

$$
\begin{gathered}
\mu_{1}=\mu \Sigma_{1} /\left(c_{2}-c_{3}\right), \quad \mu_{2}=\mu \Sigma_{2} /\left(c_{3}-c_{1}\right), \quad \mu_{3}=\mu \Sigma_{3} /\left(c_{1}-c_{2}\right), \\
\Sigma_{1}=V_{22} V_{33}-V_{32} V_{23}, \quad \Sigma_{2}=V_{32} V_{13}-V_{33} V_{12}, \quad \Sigma_{3}=V_{12} V_{23}-V_{13} V_{22},
\end{gathered}
$$

$\mu \neq 0$ being an arbitrary factor. Substituting these expressions into (24) and using the obvious identity

$$
\Sigma_{1} \mathbf{V}_{1}+\Sigma_{2} \mathbf{V}_{2}+\Sigma_{3} \mathbf{V}_{3}=0
$$

after transformations we find

$$
\begin{aligned}
& p=\frac{\mu}{\left(c_{1}-c_{2}\right)\left(c_{2}-c_{3}\right)\left(c_{3}-c_{1}\right)}\left(c_{1} \Sigma_{1} \mathbf{V}_{1}+c_{2} \Sigma_{2} \mathbf{V}_{2}+c_{3} \Sigma_{3} \mathbf{V}_{3}\right) \\
& z=\frac{\mu}{\left(c_{1}-c_{2}\right)\left(c_{2}-c_{3}\right)\left(c_{3}-c_{1}\right)}\left(c_{2} c_{3} \Sigma_{1} \mathbf{V}_{1}+c_{1} c_{3} \Sigma_{2} \mathbf{V}_{2}+c_{1} c_{2} \Sigma_{3} \mathbf{V}_{3}\right)
\end{aligned}
$$

As a result,

$$
z+s p=\frac{\mu}{\left(c_{1}-c_{2}\right)\left(c_{2}-c_{3}\right)\left(c_{3}-c_{1}\right)} \sum_{\alpha=1}^{3}\left(c_{\alpha} s+c_{\beta} c_{\gamma}\right) \Sigma_{\alpha} \mathbf{V}_{\alpha} .
$$

Now we calculate the components of $\mathbf{V}_{\alpha}$. Up to an arbitrary nonzero factor, they can be found from the system of equations

$$
V_{\alpha 1} x_{1}+V_{\alpha 2} x_{2}+V_{\alpha 3} x_{3}=0, \quad \sum_{i=1}^{3}\left(c_{\alpha}-b_{i}\right) V_{\alpha i}^{2}=0, \quad \alpha=1,2,3,
$$

which represent the conditions that the line $\ell_{\alpha}$ passes through the focus $\mathbf{P}=\left(x_{1}: x_{2}: x_{3}\right)$ and touches the quadric $Q\left(c_{\alpha}\right)$.

In the sequel we apply the normalization $x_{1}^{2}+x_{2}^{2}+x_{3}^{2}=1$, which gives rise to expressions (18).

For $\mathbf{P} \notin Q\left(c_{\alpha}\right)$, this system possesses two different solutions, and for $\mathbf{P} \in Q\left(c_{\alpha}\right)$ a single one (the line touches $Q\left(c_{\alpha}\right)$ at the point $\left.\mathbf{P}\right)$. In the latter case we can just put

$$
V_{\alpha i}=x_{i} /\left(c_{\alpha}-b_{i}\right) .
$$

Next, it is obvious that under reflection $\left(x_{1}: x_{2}: x_{3}\right) \rightarrow\left(-x_{1}: x_{2}: x_{3}\right)$, a solution $\left(V_{\alpha 1}: V_{\alpha 2}\right.$ : $\left.V_{\alpha 3}\right)$ transforms to $\left(-V_{\alpha 1}: V_{\alpha 2}: V_{\alpha 3}\right)$ (similarly, for the two other reflections). Let us seek solutions of equations (30) in the form of symmetric functions of the complex coordinates $\lambda_{1}, \lambda_{2}$ such that

1) for $\lambda_{1}=c_{\alpha}$ or $\lambda_{2}=c_{\alpha}$ (i.e., when $\mathbf{P} \in Q\left(c_{\alpha}\right)$ ) there is a unique solution proportional to (31);

2) if $\lambda_{1}$ or $\lambda_{2}$ circles around the point $\lambda=c_{\alpha}$ on the complex plane $\lambda$, the two solutions transform into each other;

3) for $\lambda_{1}=b_{i}$ or $\lambda_{2}=b_{i}$ (i.e., when $x_{i}=0$ ), $V_{\alpha i}$ does not vanishes. 
Using the Jacobi identities

$$
\sum_{i=1}^{n} \frac{a_{i}^{k}}{\prod\left(a_{i}-a_{j}\right)}=\left\{\begin{array}{l}
0, \quad k<n-1 \\
1, \quad k=n-1 \\
\sum_{i=1}^{n} a_{i}, \quad k=n
\end{array}\right.
$$

one can check that the following expressions satisfy equations (30) and the above three conditions

$$
V_{\alpha i}=x_{i}\left(\frac{\sqrt{\Phi\left(\lambda_{1}\right)\left(\lambda_{2}-c_{\alpha}\right)}}{\lambda_{1}-b_{i}}+\frac{\sqrt{\Phi\left(\lambda_{2}\right)\left(\lambda_{1}-c_{\alpha}\right)}}{\lambda_{2}-b_{i}}\right), \quad x_{i}=\frac{\sqrt{\left(\lambda_{1}-b_{i}\right)\left(\lambda_{2}-b_{i}\right)}}{\sqrt{\left(b_{i}-b_{j}\right)\left(b_{i}-b_{k}\right)}} .
$$

Then, using again the identities (32), we have

$$
\left\langle\mathbf{V}_{\alpha}, \mathbf{V}_{\beta}\right\rangle \equiv\left(\lambda_{2}-\lambda_{1}\right)\left(\sqrt{\left(\lambda_{2}-c_{\alpha}\right)\left(\lambda_{2}-c_{\beta}\right)}-\sqrt{\left(\lambda_{1}-c_{\alpha}\right)\left(\lambda_{1}-c_{\beta}\right)}\right) .
$$

and, in particular, $\left\langle\mathbf{V}_{\alpha}, \mathbf{V}_{\alpha}\right\rangle=\left(\lambda_{1}-\lambda_{2}\right)^{2}$ for $\alpha=1,2,3$.

Next, substituting (33) into (26) and applying the symbolic multiplication rule $\sqrt{a b} \sqrt{a c}=a \sqrt{b c}$, we find the factors $\Sigma_{\alpha}$ in form

$$
\begin{gathered}
\Sigma_{\alpha}=\left(\lambda_{1}-\lambda_{2}\right) x_{1}\left(\sqrt{-\left(\lambda_{1}-c_{\gamma}\right)\left(\lambda_{2}-c_{\beta}\right)}-\sqrt{-\left(\lambda_{1}-c_{\beta}\right)\left(\lambda_{2}-c_{\gamma}\right)}\right), \\
(\alpha, \beta, \gamma)=(1,2,3) .
\end{gathered}
$$

Further, putting (33), (35) into (29), we obtain

$$
\begin{aligned}
z_{i}+s p_{i} & =\frac{\mu\left(\lambda_{1}-\lambda_{2}\right) x_{1}}{\left(c_{1}-c_{2}\right)\left(c_{2}-c_{3}\right)\left(c_{3}-c_{1}\right)} x_{i} \cdot \sum_{\alpha=1}^{3}\left(c_{\alpha} s+c_{\beta} c_{\gamma}\right) \\
& \cdot\left[\frac{\sqrt{\Phi\left(\lambda_{1}\right) \psi\left(\lambda_{2}\right)}}{\lambda_{1}-b_{i}}\left(\sqrt{\frac{\lambda_{1}-c_{\gamma}}{\lambda_{2}-c_{\gamma}}}-\sqrt{\frac{\lambda_{1}-c_{\beta}}{\lambda_{2}-c_{\beta}}}\right)+\frac{\sqrt{\Phi\left(\lambda_{2}\right) \psi\left(\lambda_{1}\right)}}{\lambda_{2}-b_{i}}\left(\sqrt{\frac{\lambda_{2}-c_{\gamma}}{\lambda_{1}-c_{\gamma}}}-\sqrt{\frac{\lambda_{1}-c_{\beta}}{\lambda_{2}-c_{\beta}}}\right)\right] \\
& \equiv \mu\left(\lambda_{1}-\lambda_{2}\right) x_{1} x_{i} \sum_{\alpha=1}^{3}\left(s-c_{\alpha}\right) \frac{\sqrt{-\left(\lambda_{1}-c_{\alpha}\right)\left(\lambda_{2}-c_{\alpha}\right)}}{\left(c_{\alpha}-c_{\beta}\right)\left(c_{\alpha}-c_{\gamma}\right)}\left(\frac{\sqrt{\Phi\left(\lambda_{1}\right) \psi\left(\lambda_{2}\right)}}{\left(\lambda_{1}-b_{i}\right)\left(\lambda_{2}-c_{\alpha}\right)}-\frac{\sqrt{\Phi\left(\lambda_{2}\right) \psi\left(\lambda_{1}\right)}}{\left(\lambda_{2}-b_{i}\right)\left(\lambda_{1}-c_{\alpha}\right)}\right),
\end{aligned}
$$

which, up to multiplication by a common factor, coincides with the numerator in Kötter's formula (16).

To determine the factor $\mu$ in (29) and in (36), we apply the condition $\langle p, p\rangle=c_{0}$ which follows from (13). Then, from (27) we get

$$
\frac{c_{0}}{\mu^{2}}=\frac{\left|c_{1} \Sigma_{1} \mathbf{V}_{1}+c_{2} \Sigma_{2} \mathbf{V}_{2}+c_{3} \Sigma_{3} \mathbf{V}_{3}\right|^{2}}{\left(c_{1}-c_{2}\right)^{2}\left(c_{2}-c_{3}\right)^{2}\left(c_{3}-c_{1}\right)^{2}}
$$

Using the expressions (34), we obtain

$$
\begin{aligned}
&\left|\sum_{\alpha=1}^{3} c_{\alpha} \Sigma_{\alpha} \mathbf{V}_{\alpha}\right|^{2} \equiv \sum_{\alpha=1}^{3}\left[c_{\alpha}^{2} \Sigma_{\alpha}^{2}\left\langle\mathbf{V}_{\alpha}, \mathbf{V}_{\alpha}\right\rangle+2 c_{\beta} c_{\gamma} \Sigma_{\beta} \Sigma_{\gamma}\left\langle\mathbf{V}_{\beta}, \mathbf{V}_{\gamma}\right\rangle\right] \\
&=\left(\lambda_{1}-\lambda_{2}\right)^{3} x_{1}^{2} \sum_{\alpha=1}^{3}[ c_{\alpha}^{2}\left(\lambda_{1}-\lambda_{2}\right)\left(\sqrt{-\left(\lambda_{1}-c_{\gamma}\right)\left(\lambda_{2}-c_{\beta}\right)}-\sqrt{-\left(\lambda_{1}-c_{\beta}\right)\left(\lambda_{2}-c_{\gamma}\right)}\right)^{2} \\
&+2 c_{\beta} c_{\gamma}\left(\sqrt{-\left(\lambda_{1}-c_{\gamma}\right)\left(\lambda_{2}-c_{\beta}\right)}-\sqrt{-\left(\lambda_{1}-c_{\beta}\right)\left(\lambda_{2}-c_{\gamma}\right)}\right) \\
& \cdot\left(\sqrt{-\left(\lambda_{1}-c_{\alpha}\right)\left(\lambda_{2}-c_{\gamma}\right)}-\sqrt{-\left(\lambda_{1}-c_{\gamma}\right)\left(\lambda_{2}-c_{\alpha}\right)}\right) \\
&\left.\cdot\left(\sqrt{-\left(\lambda_{2}-c_{\beta}\right)\left(\lambda_{2}-c_{\gamma}\right)}-\sqrt{-\left(\lambda_{1}-c_{\beta}\right)\left(\lambda_{1}-c_{\gamma}\right)}\right)\right]
\end{aligned}
$$


Simplifying the above expression and again using symbolic multiplication of square roots, one can verify that it is a full square:

$$
\left|\sum_{\alpha=1}^{3} c_{\alpha} \Sigma_{\alpha} \mathbf{V}_{\alpha}\right|^{2}=x_{1}^{2}\left(\lambda_{1}-\lambda_{2}\right)^{4}\left(\sum_{\alpha=1}^{3}\left(c_{\beta}-c_{\gamma}\right) \sqrt{-\left(\lambda_{1}-c_{\alpha}\right)\left(\lambda_{2}-c_{\alpha}\right)}\right)^{2} .
$$

Hence, from (37) we find

$$
\frac{\sqrt{c_{0}}}{\mu}=x_{1}\left(\lambda_{1}-\lambda_{2}\right)^{2} \sum_{\alpha=1}^{3} \frac{\sqrt{-\left(\lambda_{1}-c_{\alpha}\right)\left(\lambda_{2}-c_{\alpha}\right)}}{\left(c_{\alpha}-c_{\beta}\right)\left(c_{\alpha}-c_{\gamma}\right)} .
$$

Combining this with (36), we finally arrive at (16).

Thus, we derived the remarkable Kötter formula by making use of the geometric interpretation of the variables $\lambda_{1}, \lambda_{2}$. We also note that the expressions (16) are symmetric in $\lambda_{1}, \lambda_{2}$.

Remark 1. As noticed above, a disordered generic pair $\left(\lambda_{1}, \lambda_{2}\right)$ gives 32 different configurations of tangent lines to the quadrics $Q\left(c_{1}\right), Q\left(c_{2}\right), Q\left(c_{3}\right)$. Since the common factor $\mu$ in (29) is defined up to sign flip, we conclude that, according to the formula (16), to each generic pair $\left(\lambda_{1}, \lambda_{2}\right)$ there correspond 64 different points $(z, p)$ on the invariant manifold (a union of D-dimensional tori) defined by the constants $c_{0}, c_{1}, c_{2}, c_{3}$. This ambiguity corresponds to different signs of the square roots in the Kötter formula.

In the next section we shall use the expressions (16) and the quadratures (20) to find explicit theta-functional solutions for the Steklov-Lyapunov systems.

\section{Explicit theta-function solution of the Steklov-Lyapunov systems}

In order to give explicit theta-functions solution, we first recall some basic formulas describing inversion of the quadratures (19). We shall mainly follow the description given in $[3,4,9]$. Consider an even order hyperelliptic Riemann surface of genus $g$ represented in the standard form

$$
\Gamma=\left\{\mu^{2}=\left(\lambda-E_{1}\right) \cdots\left(\lambda-E_{2 g+2}\right)\right\} \in \mathbb{C}^{2}(\lambda, \mu) .
$$

In the sequel we shall regard $\Gamma$ as its complex compactification obtained by gluing two infinite points $\infty_{-}, \infty_{+}$, where the coordinate $\lambda$ equals infinity.

Consider also differential 1-form (differential) $\omega=\phi(\tau) d \tau$ on $\Gamma$, where $\tau$ is a local parameter at a point $P \in \Gamma$. A differential $\omega$ is called holomorphic if $\phi(\tau)$ is a holomorphic function for any point $P$. We choose the canonical basis of cycles $\mathfrak{a}_{1}, \ldots, \mathfrak{a}_{g}, \mathfrak{b}_{1}, \ldots, \mathfrak{b}_{g}$ on the surface $\Gamma$ such that their intersections are of the form:

$$
\mathfrak{a}_{i} \circ \mathfrak{a}_{j}=\mathfrak{b}_{i} \circ \mathfrak{b}_{j}=0, \quad \mathfrak{a}_{i} \circ \mathfrak{b}_{j}=\delta_{i j}, \quad i, j=1, \ldots, g,
$$

where $\gamma_{1} \circ \gamma_{2}$ denotes the intersection index of the cycles $\gamma_{1}, \gamma_{2}$.

An example of a canonical basis of cycles on $\Gamma$ is shown on Figure 2. The parts of the cycles on the lower sheet are shown by dashed lines. that

Next, let $\bar{\omega}_{1}, \ldots, \bar{\omega}_{g}$ be the conjugated basis of normalized holomorphic differentials on $\Gamma$ such

$$
\oint_{\mathfrak{a}_{j}} \bar{\omega}_{i}=2 \pi \jmath \delta_{i j}, \quad \jmath=\sqrt{-1} .
$$

The $g \times g$ matrix of $b$-periods $B_{i j}=\oint_{\mathfrak{b}_{j}} \bar{\omega}_{i}$ is symmetric and has a negative definite real part. Consider the period lattice $\Lambda^{0}=\left\{2 \pi j \mathbb{Z}^{g}+B \mathbb{Z}^{g}\right\}$ of rank $2 g$ in $\mathbb{C}^{g}=\left(z_{1}, \ldots, z_{g}\right)$. The complex torus $\operatorname{Jac}(G)=\mathbb{C}^{g} / \Lambda^{0}$ is called the Jacobi variety (Jacobian) of the curve $G$. 


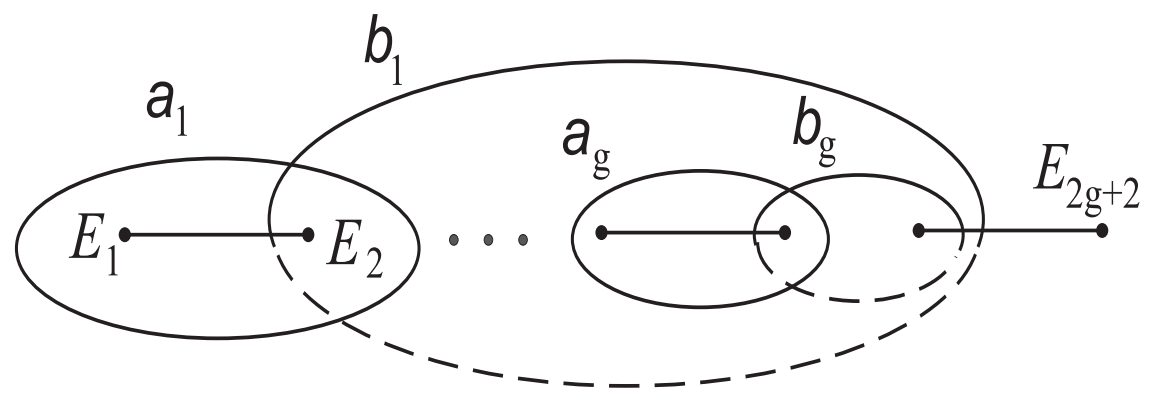

Figure 2:

Now consider a generic divisor of points $P_{1}=\left(\lambda_{1}, \mu_{1}\right), \ldots, P_{g}=\left(\lambda_{g}, \mu_{g}\right)$ on it, and the AbelJacobi mapping with a basepoint $P_{0}$

$$
\begin{gathered}
\int_{P_{0}}^{P_{1}} \bar{\omega}+\cdots+\int_{P_{0}}^{P_{g}} \bar{\omega}=z, \\
\bar{\omega}=\left(\bar{\omega}_{1}, \ldots, \bar{\omega}_{g}\right)^{T}, \quad z=\left(z_{1}, \ldots, z_{g}\right)^{T} \in \mathbb{C}^{g} .
\end{gathered}
$$

Under the mapping, functions on $S^{g} \Gamma$, i.e., symmetric functions of the points $P_{1}, \ldots, P_{g}$ are $2 g$-fold periodic functions of the complex variables $z_{1}, \ldots, z_{g}$ with the above period lattice $\Lambda^{0}$ (Abelian functions).

Explicit expressions of such functions can be obtained by means of theta-functions on the universal covering $\mathbb{C}^{g}=\left(z_{1}, \ldots, z_{g}\right)$ of the complex torus. Recall that customary Riemann's theta-function $\theta(z \mid B)$ associated with the Riemann matrix $B$ is defined by the series ${ }^{2}$

$$
\begin{gathered}
\theta(z \mid B)=\sum_{M \in \mathbb{Z}^{g}} \exp (\langle B M, M\rangle+\langle M, z\rangle), \\
\langle M, z\rangle=\sum_{i=1}^{g} M_{i} z_{i}, \quad\langle B M, M\rangle=\sum_{i, j=1}^{g} B_{i j} M_{i} M_{j} .
\end{gathered}
$$

Equation $\theta(z \mid B)=0$ defines a codimension one subvariety $\Theta \in \operatorname{Jac}(\Gamma)$ (for $g>2$ with singularities) called theta-divisor.

We shall also use theta-functions with characteristics $\alpha=\left(\alpha_{1}, \ldots, \alpha_{g}\right), \beta=\left(\beta_{1}, \ldots, \beta_{g}\right), \alpha_{j}, \beta_{j} \in$ $\mathbb{R}$, which are obtained from $\theta(z \mid B)$ by shifting the argument $z$ and multiplying by an exponent ${ }^{3}$ :

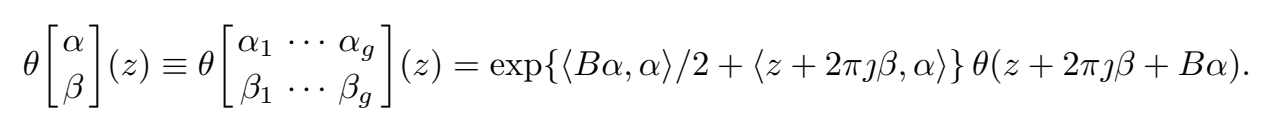

All these functions enjoy the quadiperiodic property

$$
\begin{array}{r}
\theta\left[\begin{array}{l}
\alpha \\
\beta
\end{array}\right](z+2 \pi \jmath K+B M)=\exp (2 \pi \jmath \epsilon) \exp \{-\langle B M, M\rangle / 2-\langle M, z\rangle\} \theta\left[\begin{array}{l}
\alpha \\
\beta
\end{array}\right](z), \\
\epsilon=\langle\alpha, K\rangle-\langle\beta, M\rangle,
\end{array}
$$

An important particular case is represented by theta-functions with half-integer characteristics

$$
\Delta=\left(\begin{array}{c}
\Delta^{\prime} \\
\Delta^{\prime \prime}
\end{array}\right), \quad \eta_{i}=\left(\begin{array}{c}
\eta_{i}^{\prime} \\
\eta_{i}^{\prime \prime}
\end{array}\right), \quad \text { and } \quad \eta_{i j}=\eta_{i}+\eta_{j} \quad\left(\bmod \mathbb{Z}^{2 g}\right), \quad \Delta^{\prime}, \Delta^{\prime \prime}, \eta_{i}^{\prime}, \eta_{i}^{\prime \prime} \in \frac{1}{2} \mathbb{Z}^{g} / \mathbb{Z}^{g}
$$

\footnotetext{
${ }^{2}$ The expression for $\theta(z)$ we use here is different from that chosen in a series of books on theta-functions by multiplication of $z$ by a constant factor.

${ }^{3}$ Here and below we omit $B$ in the theta-functional notation.
} 
such that

$$
\begin{aligned}
2 \pi \jmath \eta_{i}^{\prime \prime}+B \eta_{i}^{\prime} & =\int_{E_{2 g+2}}^{E_{i}} \bar{\omega}(\bmod \Lambda), \\
2 \pi \jmath \Delta^{\prime \prime}+B \Delta^{\prime} & =\mathcal{K}(\bmod \Lambda),
\end{aligned}
$$

$\mathcal{K} \in \mathbb{C}^{g}$ being the vector of the Riemann constants and $E_{i}$ briefly denotes the branch point $\left(E_{i}, 0\right)$ on $\Gamma$.

For the case $g=2$ and for the chosen canonical basis of cycles $\mathfrak{a}_{1}, \mathfrak{a}_{2}, \mathfrak{b}_{1}, \mathfrak{b}_{2}$ on $\Gamma$ the above characteristics $\Delta, \eta_{i}$ are

$$
\begin{array}{rlrl}
\Delta & =\left(\begin{array}{cc}
1 / 2 & 1 / 2 \\
0 & 1 / 2
\end{array}\right), & \eta_{1}=\left(\begin{array}{cc}
1 / 2 & 0 \\
0 & 0
\end{array}\right), \quad \eta_{2}=\left(\begin{array}{cc}
1 / 2 & 0 \\
1 / 2 & 0
\end{array}\right), \\
\eta_{3}=\left(\begin{array}{cc}
0 & 1 / 2 \\
1 / 2 & 0
\end{array}\right), & \eta_{4}=\left(\begin{array}{cc}
0 & 1 / 2 \\
1 / 2 & 1 / 2
\end{array}\right), \quad \eta_{5}=\left(\begin{array}{cc}
0 & 0 \\
1 / 2 & 1 / 2
\end{array}\right),
\end{array}
$$

and, by convention, $\eta_{6}$ is the zero theta-characteristic. Note also the property

$$
\eta_{1}+\eta_{3}+\eta_{5}=\eta_{2}+\eta_{4}=\Delta .
$$

The root functions. To obtain theta-functions solution for many problems linearized on Jacobians of hyperelliptic curves, one can apply some remarkable relations between roots of certain functions on symmetric products of such curves and quotients of theta-functions with half-integer characteristics, which are historically referred to as root functions. For the case of odd order hyperelliptic curves such functions were obtained by Weierstrass and Rosenheim [19, 13], see also $[3,4]$.

For our purposes it is sufficient to quote only several root functions for the particular case $g=2$ and the even-order hyperelliptic curve

$$
\Gamma=\left\{\mu^{2}=R(\lambda)\right\}, \quad R(\lambda)=\left(\lambda-E_{1}\right) \cdots\left(\lambda-E_{6}\right) .
$$

Let us introduce the polinomial $U(\lambda, s)=\left(s-\lambda_{1}\right)\left(s-\lambda_{2}\right)$.

Proposition 3. Under the Abel-Jacobi mapping (38) with $g=2$ and the basepoint $P_{0}=E_{6}$ the following relations hold

$$
\begin{gathered}
U\left(\lambda, E_{i}\right) \equiv\left(\lambda_{1}-E_{i}\right)\left(\lambda_{2}-E_{i}\right)=\kappa_{i} \frac{\theta^{2}\left[\Delta+\eta_{i}\right](z)}{\theta[\Delta](z-q / 2) \theta[\Delta](z+q / 2)}, \\
q=\int_{\infty_{-}}^{\infty_{+}} \bar{\omega}=2 \int_{E_{6}}^{\infty_{+}} \bar{\omega}, \quad \kappa_{i}=\text { const, } \quad i=1, \ldots, 6, \\
\frac{1}{\lambda_{1}-\lambda_{2}}\left(\frac{\sqrt{R\left(\lambda_{1}\right)}}{\left(E_{i}-\lambda_{1}\right)\left(E_{j}-\lambda_{1}\right)\left(E_{s}-\lambda_{1}\right)}-\frac{\sqrt{R\left(\lambda_{2}\right)}}{\left(E_{i}-\lambda_{2}\right)\left(E_{j}-\lambda_{2}\right)\left(E_{s}-\lambda_{2}\right)}\right) \\
=\kappa_{i j s} \frac{\theta\left[\Delta+\eta_{i}+\eta_{j}+\eta_{s}\right](z) \theta[\Delta](z-q / 2) \theta[\Delta](z+q / 2)}{\theta\left[\Delta+\eta_{i}\right](z) \theta\left[\Delta+\eta_{j}\right](z) \theta\left[\Delta+\eta_{s}\right](z)} \\
\frac{\sqrt{R\left(\lambda_{1}\right)}}{R\left(E_{i}\right)} \sqrt{U\left(\lambda, E_{j}\right)} \\
\left(\frac{\sqrt{R\left(\lambda_{2}\right)}}{\left(E_{i}-\lambda_{1}\right)\left(E_{j}-\lambda_{1}\right)\left(E_{s}-\lambda_{1}\right)}-\frac{\theta}{\left(E_{i}-\lambda_{2}\right)\left(E_{j}-\lambda_{2}\right)\left(E_{s}-\lambda_{2}\right)}\right) \\
=\kappa_{i j s}^{\prime} \frac{\theta\left[\Delta+\eta_{i}+\eta_{j}+\eta_{s}\right](z)}{\theta\left[\Delta+\eta_{s}\right](z)}, \\
\kappa_{i j s}, \kappa_{i j s}^{\prime}=\text { const, } \quad i, j, s=1, \ldots, 6, \quad i \neq j \neq s \neq i,
\end{gathered}
$$

where, as above, $\eta_{6}$ is the zero theta-characteristic and $\infty_{+}, \infty_{-}$are the infinite points of the compactified curve $\Gamma$. The constant factors $\kappa_{i}, \kappa_{i j s}, \kappa_{i j s}^{\prime}$ depend on the moduli of $\Gamma$ only. 
Sketch of proof of Proposition 3. The left and right hand sides of (44) are meromorphic functions on $\operatorname{Jac}(\Gamma)$, which have the same zeros and poles with the same multiplicity. This implies that their quotient is an analytic function on a compact complex manifold without poles and therefore a constant.

The root functions (45), (46) can be deduced from the corresponding root functions for the case of odd-order hyperelliptic curve, by making a fractionally-linear transformation of $\lambda$ that sends the Weierstrass point $E_{2 g+2}$ on $\Gamma$ to infinity.

The constants $\kappa_{i}, \kappa_{i j s}, \kappa_{i j s}^{\prime}$ can be calculated explicitly in terms of the coordinates $E_{1}, \ldots, E_{6}$ and theta-constants by equating $\lambda_{1}, \lambda_{2}$ to certain $E_{i}$ and the argument $z$ to the corresponding half-period in $\operatorname{Jac}(\Gamma)$ (see, e.g., [3]).

Explicit solution. Now we are able to write explicit solution for the Steklov-Lyapunov systems by comparing the root functions (44), (46) with the Kötter expression (16).

Namely, let $\Gamma=\left\{\mu^{2}=\Phi(\lambda) \varphi(\lambda)\right\}$ where the polynomials $\phi$ and $\varphi$ are defined in (17) and identify (without ordering) the sets

$$
\left\{E_{1}, E_{2}, E_{3}, E_{4}, E_{5}, E_{6}\right\}=\left\{b_{1}, b_{2}, b_{3}, c_{1}, c_{2}, c_{3}\right\} .
$$

By $\eta_{b_{i}}, \eta_{c_{\alpha}}$ we denote the half-integer characteristics corresponding to the branch points $\left(b_{i}, 0\right),\left(c_{\alpha}, 0\right)$ respectively, according to formula (41).

Theorem 4. For fixed constants of motion $c_{1}, c_{2}, c_{3}$ the variables $z, p$ can be expressed in terms of theta-functions of the curve $\Gamma$ in a such a way that for any $s \in \mathbb{C}$

$$
z_{i}+s p_{i}=\frac{\sum_{\alpha=1}^{3} k_{i \alpha}\left(s-c_{\alpha}\right) \theta\left[\Delta+\eta_{c_{\beta}}+\eta_{c_{\gamma}}+\eta_{b_{i}}\right](z)}{\sum_{\alpha=1}^{3} k_{0 \alpha} \theta\left[\Delta+\eta_{c_{\alpha}}\right](z)}, \quad(\alpha, \beta, \gamma)=(1,2,3),
$$

where $k_{i \alpha}, k_{0 \alpha}$ are certain constants depending on the moduli of $\Gamma$ only, and the components of the argument $z$ are linear functions of $t$ :

$$
z_{j}=C_{j 1} \delta_{1}+C_{j 2} \delta_{2}+z_{j 0}, \quad z_{j 0}=\text { const }, \quad C=A^{-1}
$$

$A$ being is the matrix of $\mathfrak{a}$-periods of the differentials $d \lambda / \mu, \lambda d \lambda / \mu$ on $\Gamma$.

Thus, we have recovered the theta-function solution of the systems obtained by Kötter in [14].

Remark 2. In view of the definition of theta-function with characteristics, under the argument shift $z \rightarrow z-\mathcal{K}$ the special characteristic $\Delta$ is killed and the solutions (47) are simplified to

$$
z_{i}+s p_{i}=\frac{\sum_{\alpha=1}^{3} \bar{k}_{i \alpha}\left(s-c_{\alpha}\right) \theta\left[\eta_{c_{\beta}}+\eta_{c_{\gamma}}+\eta_{b_{i}}\right](z)}{\sum_{\alpha=1}^{3} \bar{k}_{0 \alpha} \theta\left[\eta_{c_{\alpha}}\right](z)}, \quad(\alpha, \beta, \gamma)=(1,2,3),
$$

where the constants $\bar{k}_{i \alpha}, \bar{k}_{0 \alpha}$ coincide with $k_{i \alpha}, k_{0 \alpha}$ in (47) up to multiplication by a quartic root of unity. In each concrete case of position of $b_{i}, c_{\alpha}$, one can also simplify the sums of characteristics in the numerator of (49) by using the relations (43).

Proof of Theorem 4. The summands in the numerator of the Kötter solution (16), when divided by $\lambda_{1}-\lambda_{2}$, can be written as

$$
\begin{gathered}
\frac{s-c_{\alpha}}{\left(c_{\alpha}-c_{\beta}\right)\left(c_{\alpha}-c_{\gamma}\right)} \frac{\sqrt{-\left(\lambda_{1}-c_{\alpha}\right)\left(\lambda_{2}-c_{\alpha}\right)}}{\lambda_{1}-\lambda_{2}} \cdot\left(\frac{\sqrt{\Phi\left(\lambda_{1}\right) \psi\left(\lambda_{2}\right)}}{\left(\lambda_{1}-b_{i}\right)\left(\lambda_{2}-c_{\alpha}\right)}-\frac{\sqrt{\Phi\left(\lambda_{2}\right) \psi\left(\lambda_{1}\right)}}{\left(\lambda_{2}-b_{i}\right)\left(\lambda_{1}-c_{\alpha}\right)}\right) \\
=\frac{s-c_{\alpha}}{\left(c_{\alpha}-c_{\beta}\right)\left(c_{\alpha}-c_{\gamma}\right)} \frac{\sqrt{-\left(\lambda_{1}-c_{\beta}\right)\left(\lambda_{2}-c_{\beta}\right)} \sqrt{-\left(\lambda_{1}-c_{\gamma}\right)\left(\lambda_{2}-c_{\gamma}\right)}}{\lambda_{1}-\lambda_{2}} \\
\times\left(\frac{\mu_{1}}{\left(\lambda_{1}-b_{i}\right)\left(\lambda_{1}-c_{\beta}\right)\left(\lambda_{1}-c_{\gamma}\right)}-\frac{\mu_{2}}{\left(\lambda_{2}-b_{i}\right)\left(\lambda_{2}-c_{\beta}\right)\left(\lambda_{2}-c_{\gamma}\right)}\right), \\
\mu_{1}=\sqrt{\Phi\left(\lambda_{1}\right) \psi\left(\lambda_{1}\right)}, \quad \mu_{2}=\sqrt{\Phi\left(\lambda_{2}\right) \psi\left(\lambda_{2}\right)} .
\end{gathered}
$$


The right hand sides have the form of the root function (46). Hence, up to a constant factor, they are equal to

$$
\left(s-c_{\alpha}\right) \frac{\theta\left[\Delta+\eta_{c_{\beta}}+\eta_{c_{\gamma}}+\eta_{b_{i}}\right](z)}{\theta\left[\Delta+\eta_{b_{i}}\right](z)} .
$$

Next, in view of (44), we obtain

$$
\begin{aligned}
& x_{i}=\varkappa_{i} \frac{\theta\left[\Delta+\eta_{b_{i}}\right](z)}{\sqrt{\theta[\Delta](z-q / 2) \theta[\Delta](z+q / 2)}}, \\
& \sqrt{-\left(\lambda_{1}-c_{\alpha}\right)\left(\lambda_{2}-c_{\alpha}\right)}=\varkappa_{\alpha} \frac{\theta\left[\Delta+\eta_{c_{\alpha}}\right](z)}{\sqrt{\theta[\Delta](z-q / 2) \theta[\Delta](z+q / 2)}}, \\
& \varkappa_{i}, \varkappa_{\alpha}=\text { const. }
\end{aligned}
$$

Combining the above expressions, we rewrite the right hand side of (16) in the form

$$
\sqrt{c_{0}} \frac{\frac{\theta\left[\Delta+\eta_{b_{i}}\right](z)}{\theta[\Delta](z-q / 2) \theta[\Delta](z+q / 2)} \sum_{\alpha=1}^{3} \frac{k_{i \alpha}\left(s-c_{\alpha}\right) \theta\left[\Delta+\eta_{c_{\beta}}+\eta_{c_{\gamma}}+\eta_{b_{i}}\right](z)}{\theta\left[\Delta+\eta_{b_{i}}\right](z)}}{\sum_{\alpha=1}^{3} \frac{k_{0 \alpha} \theta\left[\Delta+\eta_{c_{\alpha}}\right](z)}{\theta[\Delta](z-q / 2) \theta[\Delta](z+q / 2)}},
$$

which, after simplifications, gives (47).

Formulas (48) follow from the relation $\left(\bar{\omega}_{1}, \bar{\omega}_{2}\right)^{T}=C(d \lambda / \mu, \lambda d \lambda / \mu)$, where, as above, $\bar{\omega}_{j}$ are the normalized holomorphic differentials on $\Gamma$, and the functions $(21)$.

\section{The pole divisor and the alternative theta-solution.}

In view of the quasiperiodic property (40), when the complex argument $z$ changes by a period vector in $\operatorname{Jac}(\Gamma)$, the theta-functions in $(47),(49)$ are multiplied by factors, which may be different. Hence, the variables $z_{i}, p_{i}$ cannot be single valued on the Jacobian variety $\Gamma$, and one can show that they are meromorphic on $\mathcal{A}$, the 16-fold unramified covering of it, obtained by doubling of all the four period vectors in $\operatorname{Jac}(\Gamma)$. This implies that $\mathcal{A}$ is also a principally polarized Abelian variety isomorphic to $\operatorname{Jac}(\Gamma)$. As follows from the structure of (47), all $z_{i}, p_{i}$ have a common set of simple poles (so called pole divisor), which we denote $\mathcal{D} \subset \mathcal{A}$.

For each $\alpha$, the zeros of $\theta\left[\eta_{c_{\alpha}}\right](z)$ in $\operatorname{Jac}(\Gamma)$ form a translate $\Theta_{\alpha}$ of the theta-divisor $\Theta$ by a halfperiod. Each translate passes via six half-periods, and $\Theta_{1}, \Theta_{2}, \Theta_{3}$ have a unique common intersection in the origin (neutral point) $\mathcal{O} \in \operatorname{Jac}(\Gamma)$. This is depicted in Fig. 3 (a), where $\Theta_{\alpha}$ are shown as circles and the half-periods in $\operatorname{Jac}(\Gamma)$ as black dots. Hence, at $z=\mathcal{O}$ the denominator of (49) vanishes. Then, under the covering $\pi: \mathcal{A} \rightarrow \operatorname{Jac}(\Gamma)$, the preimage of $\mathcal{O}$ consists of all the 16 half-periods in $\mathcal{A}$, which therefore belong to the divisor $\mathcal{D}$.

On the other hand, as was shown in $[1,2]$ by applying the Kovalevskaya-Painlevé analysis to the integrable flow on the algebra $s o(4)$ with the diagonal metric II ${ }^{4}$, the pole divisor $\mathcal{D}$ is a union of 4 translates $\mathcal{D}_{j}, j=0,1,2,3$ of the genus 2 curve $\Gamma$ embedded into $\mathcal{A}$, which are obtained from each other by shifting by certain half-periods in $\mathcal{A}$.

The union passes through all the 16 half-periods in $\mathcal{A}$ according to the intersection pattern shown in Fig. 3 (b), which we borrowed from [2]. Here the circles represent the translates $\mathcal{D}_{j}$ and the 16 black dots depict the half-periods. Under the projection $\pi$ all the above half-periods are mapped onto $\mathcal{O} \in \operatorname{Jac}(\Gamma)$.

Now assume again that $b_{1}<b_{2}<b_{3}$.

Proposition 5. The four translates $\mathcal{D}_{j}$ are obtained from each other by shifts in $\mathcal{A}$ by the half-periods with the characteristics $\eta_{1}, \bar{\eta}$, and $\eta_{1}+\bar{\eta}$, where, if all $c_{\alpha}$ are real,

$$
\bar{\eta}=\eta_{4} \text { if } \quad b_{1}<c_{1}<c_{2}<b_{2}<c_{3}<b_{3}, \quad \bar{\eta}=\eta_{3} \text { if } \quad b_{1}<c_{1}<b_{2}<c_{2}<c_{3}<b_{3},
$$

\footnotetext{
${ }^{4} \mathrm{As}$ we mentioned in Introduction, there is a linear isomorphism between this integrable flow on $s o(4)$ and the Steklov-Lyapunov systems, hence the result of $[1,2]$ about $\mathcal{D}$ equally holds for the latter systems.
} 


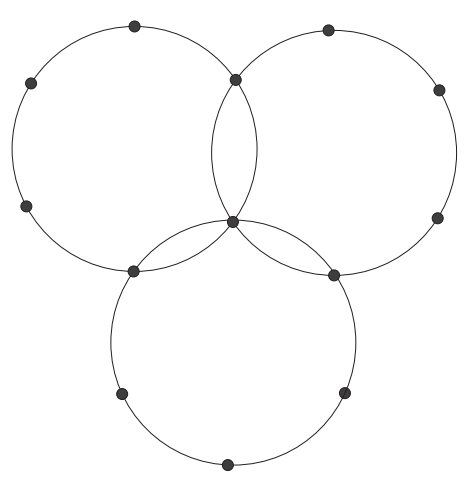

(a)

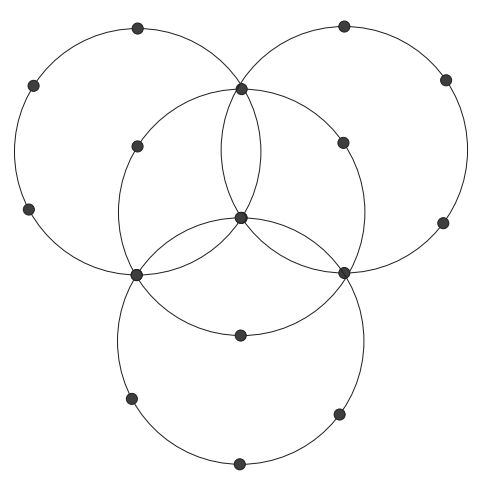

(b)

Figure 3: (a) Configuration of the translates $\Theta_{\alpha}$ in $\operatorname{Jac}(\Gamma)$. (b) The 4 translates of $\Gamma$ in $\mathcal{A}$ forming the pole divisor $\mathcal{D}$.

which in both cases gives $\bar{\eta}=\eta_{b_{2}}$. If $c_{1}$ is real and $c_{2}, c_{3}$ are complex conjugated, then

$$
\bar{\eta}=\eta_{2} \text { if } \quad c_{1}<\Re c_{2}, \quad \bar{\eta}=\eta_{5} \text { if } \Re c_{2}<c_{1} .
$$

In all the cases one can choose $\eta_{1}=\eta_{b_{1}}$.

Proof. Choose a generic point $q \in \mathcal{D}$ and let $z^{*}$ be its projection onto $\operatorname{Jac}(\Gamma)$ such that

$$
f\left(z^{*}\right)=\sum_{\alpha=1}^{3} \bar{k}_{\alpha} \theta\left[\eta_{c_{\alpha}}\right]\left(z^{*}\right)=0 \quad \text { and } \quad \theta\left[\eta_{c_{\alpha}}\right]\left(z^{*}\right) \neq 0 \quad \text { for } \quad \alpha=1,2,3 \text {. }
$$

There exists a unique lattice $L_{2}=\left\{\mathbb{Z} \mathcal{V}_{1}+\mathbb{Z} \mathcal{V}_{2}\right\}$ generated by 2 independent period vectors $\mathcal{V}_{1}+\mathbb{Z} \mathcal{V}_{2}$ in $\operatorname{Jac}(\Gamma)$ such that under translations $z^{*} \rightarrow z^{*}+M \mathcal{V}_{1}+N \mathcal{V}_{2}, M, N \in \mathbb{Z}$ all the functions $\theta\left[\eta_{c_{\alpha}}\right]\left(z^{*}\right)$ are multiplied by the same factor and therefore $f\left(z^{*}+M \mathcal{V}_{1}+N \mathcal{V}_{2}\right)=0$. Indeed, in view of the quasi-periodicity of $\theta\left[\eta_{c_{\alpha}}\right](z)$ and the relation (50), the latter happens if and only if one of the points $\left(\lambda_{1}, \mu_{1}\right),\left(\lambda_{2}, \mu_{2}\right)$ on $\Gamma$ makes a cycle, whose projection onto the $\lambda$-plane either embraces once all the Weierstrass points $\left(c_{1}, 0\right),\left(c_{2}, 0\right),\left(c_{3}, 0\right)$ or embraces non of them. Under the Abel map, the first option leads to the shift in $\operatorname{Jac}(\Gamma)$ by the period $\mathcal{V}_{1}=4 \pi \eta_{1}^{\prime \prime}+2 B \eta_{1}^{\prime}$ and the second option to the shift by $\mathcal{V}_{2}=4 \pi \bar{\eta}^{\prime \prime}+2 B \bar{\eta}^{\prime}$.

Since the shifts of $L_{2}$ correspond to translations in $\mathcal{A}$ by the half-periods $\mathcal{V}_{1} / 2, \mathcal{V}_{2} / 2,\left(\mathcal{V}_{1}+\mathcal{V}_{2}\right) / 2$, we conclude that the divisor $\mathcal{D}$ is invariant under the above translations.

Let us now choose the origin of $\mathcal{A}$ at one of the four triple intersections of $\mathcal{D}_{j}$. Then identify the components $\mathcal{D}_{0}, \mathcal{D}_{1}, \mathcal{D}_{2}, \mathcal{D}_{3}$ as zeros of the functions

$$
\theta[\Delta](z / 2 \mid B), \quad \theta\left[\Delta+\eta_{1}\right](z / 2 \mid B), \quad \theta[\Delta+\bar{\eta}](z / 2 \mid B), \quad \theta\left[\Delta+\eta_{1}+\bar{\eta}\right](z / 2 \mid B),
$$

which we denote for brevity as $\theta_{0}(z / 2), \theta_{1}(z / 2), \theta_{2}(z / 2), \theta_{3}(z / 2)$. Here, as above, $B$ is the Riemann matrix of $\Gamma$ and $z \in \mathbb{C}^{2}$ is defined by (48). As a result, the denominator of the solution (49) has the same zeros as the theta product $\theta_{0}(z / 2) \theta_{1}(z / 2) \theta_{2}(z / 2) \theta_{3}(z / 2)$, that is, a theta-function of 4 th order on $\mathcal{A}$. The action of the translations by the half-periods $\mathcal{V}_{1} / 2, \mathcal{V}_{2} / 2, \mathcal{V}_{1} / 2+\mathcal{V}_{2} / 2$ in $\mathcal{A}$ on the components $\left(\mathcal{D}_{0}, \mathcal{D}_{1}, \mathcal{D}_{2}, \mathcal{D}_{3}\right)$ gives respectively

$$
\left(\mathcal{D}_{1}, \mathcal{D}_{0}, \mathcal{D}_{3}, \mathcal{D}_{2}\right), \quad\left(\mathcal{D}_{2}, \mathcal{D}_{3}, \mathcal{D}_{0}, \mathcal{D}_{1}\right), \quad\left(\mathcal{D}_{3}, \mathcal{D}_{2}, \mathcal{D}_{1}, \mathcal{D}_{0}\right)
$$

Solutions for the variables $v_{k}$. Note that theta-function solutions for the new phase variables $v_{1}, \ldots, v_{6}$ introduced in (11) have a more compact form. Namely, as follows from expressions (49), the functions $v_{1}+v_{2}$ and $v_{1}-v_{2}$ can have only simple poles at most along the components of the 
divisor $\mathcal{D}$. On the other hand, the integrals (12) imply the following remarkable property: the poles (the zeros) of $v_{1}+v_{2}$ are the zeros (resp. the poles) of $v_{1}-v_{2}$. Since both functions are meromorphic on $\mathcal{A}$, this necessarily implies that $v_{1}+v_{2}$ has poles along two certain components $\mathcal{D}_{j_{1}}, \mathcal{D}_{j_{2}}$ and zeros along the other two components $\mathcal{D}_{j_{3}}, \mathcal{D}_{j_{4}}$, and vice versa for $v_{1}-v_{2}$. (Note that neither $v_{1}+v_{2}$ nor $v_{1}-v_{2}$ can have simple poles along only one component $\mathcal{D}_{j}$, since in that case these functions will not be meromorphic on $\mathcal{A}$.)

The same observations hold for the pairs $\left(v_{3}+v_{4}, v_{3}-v_{4}\right)$ and $\left(v_{5}+v_{6}, v_{5}-v_{6}\right)$. Note also that functions from different pairs cannot have the same poles, since in that case they would also have the same zeros and their quotient would be constant, which is not true.

Now let us fix the origin of $\mathcal{A}$ at one specific triple intersection of $\mathcal{D}_{j}$ such that the 3 functions $v_{1}+v_{2}, v_{3}+v_{4}, v_{5}+v_{6}$ have a common pole along $\mathcal{D}_{0}$. In this case the following proposition holds.

Proposition 6. The theta-function solutions for the variables $v_{k}$ have the form

$$
\begin{gathered}
v_{1}+v_{2}=\chi_{1} \frac{\theta_{1}(z / 2) \theta_{2}(z / 2)}{\theta_{0}(z / 2) \theta_{3}(z / 2)}, \quad v_{1}-v_{2}=\chi_{2} \frac{\theta_{0}(z / 2) \theta_{3}(z / 2)}{\theta_{1}(z / 2) \theta_{2}(z / 2)}, \\
v_{3}+v_{4}=\chi_{3} \frac{\theta_{2}(z / 2) \theta_{3}(z / 2)}{\theta_{0}(z / 2) \theta_{1}(z / 2)}, \quad v_{3}-v_{4}=\chi_{4} \frac{\theta_{0}(z / 2) \theta_{1}(z / 2)}{\theta_{2}(z / 2) \theta_{3}(z / 2)}, \\
v_{5}+v_{6}=\chi_{5} \frac{\theta_{1}(z / 2) \theta_{3}(z / 2)}{\theta_{0}(z / 2) \theta_{2}(z / 2)}, \quad v_{5}-v_{6}=\chi_{6} \frac{\theta_{0}(z / 2) \theta_{2}(z / 2)}{\theta_{1}(z / 2) \theta_{3}(z / 2)}, \\
\chi_{1}, \chi_{3}, \chi_{5}=\mathrm{const}, \quad \chi_{2}=\frac{\psi\left(b_{1}\right)}{\left(b_{2}-b_{3}\right) \chi_{1}}, \quad \chi_{4}=\frac{\psi\left(b_{2}\right)}{\left(b_{3}-b_{1}\right) \chi_{3}}, \quad \chi_{6}=\frac{\psi\left(b_{3}\right)}{\left(b_{1}-b_{2}\right) \chi_{5}} .
\end{gathered}
$$

Proof. As follows from the Kötter formula (16) and theta-solutions (49), the translations by the period vectors $\mathcal{V}_{1}, \mathcal{V}_{2}, \mathcal{V}_{1}+\mathcal{V}_{2}$ in $\operatorname{Jac}(\Gamma)$ generate the involutions

$$
\begin{aligned}
& \sigma_{1}:\left(z_{1}, p_{1}, z_{2}, p_{2}, z_{3}, p_{3}\right) \mapsto\left(z_{1}, p_{1},-z_{2},-p_{2}, z_{3}, p_{3}\right), \\
& \sigma_{2}:\left(z_{1}, p_{1}, z_{2}, p_{2}, z_{3}, p_{3}\right) \mapsto\left(-z_{1},-p_{1},-z_{2},-p_{2}, z_{3}, p_{3}\right), \\
& \sigma_{3}=\sigma_{2} \circ \sigma_{1}:\left(z_{1}, p_{1}, z_{2}, p_{2}, z_{3}, p_{3}\right) \mapsto\left(-z_{1},-p_{1}, z_{2}, p_{2}, z_{3}, p_{3}\right),
\end{aligned}
$$

which, in view of (11), gives rise to the transformations

$$
\begin{array}{llll}
\sigma_{1}: & v_{2}+v_{1} \longleftrightarrow v_{2}-v_{1}, & v_{4} \pm v_{3} \longleftrightarrow v_{4} \pm v_{3}, & v_{5}+v_{6} \longleftrightarrow v_{5}-v_{6}, \\
\sigma_{2}: & v_{2}+v_{1} \longleftrightarrow v_{2}-v_{1}, & v_{4}+v_{3} \longleftrightarrow v_{4}-v_{3}, & v_{5} \pm v_{6} \longleftrightarrow-\left(v_{5} \pm v_{6}\right) \\
\sigma_{3}: & v_{2} \pm v_{1} \longleftrightarrow v_{2} \pm v_{1}, & v_{4}+v_{3} \longleftrightarrow v_{4}-v_{3}, & v_{6}+v_{5} \longleftrightarrow v_{6}-v_{5} .
\end{array}
$$

Now observe that under the action of $\sigma_{i}$ on the left-hand sides of the relations (53) and the corresponding transformation of theta-functions (51) under the action (52), these relations transform to each other. Moreover, one can check that the left- and right hand sides of (53) are multiplied by the same factors under the shift of $z$ by any period vector of $\operatorname{Jac}(\Gamma)$. This, together with the above observations on the poles and zeros of $v_{1}+v_{2}, v_{1}-v_{2}$, etc, proves (53).

The relations (54) between the constants $\chi_{i}$ follow from the first 3 integrals in (12).

The constants $\chi_{1}, \chi_{2}, \chi_{3}$ can be calculated explicitly in terms of $b_{i}, c_{\alpha}$ and theta-constants of $\Gamma$.

As follows from the solutions (53), the product $\left(v_{1}+v_{2}\right)\left(v_{3}+v_{4}\right)$ and the other two similar products have double poles along $\mathcal{D}_{0}$ only:

$$
\begin{gathered}
\left(v_{1}+v_{2}\right)\left(v_{3}+v_{4}\right)=g_{2} \frac{\theta_{2}^{2}(z / 2)}{\theta_{0}^{2}(z / 2)}, \\
\left(v_{3}+v_{4}\right)\left(v_{5}+v_{6}\right)=g_{3} \frac{\theta_{3}^{2}(z / 2)}{\theta_{0}^{2}(z / 2)}, \quad\left(v_{1}+v_{2}\right)\left(v_{5}+v_{6}\right)=g_{1} \frac{\theta_{1}^{2}(z / 2)}{\theta_{0}^{2}(z / 2)}, \\
g_{1}, g_{2}, g_{3}=\text { const. }
\end{gathered}
$$

Analogs of these expressions were obtained in paper [8] in relation with separation of variables for the integrable system on $s o(4)$ with the diagonal metric II. Due to the linear isomorphism between this system and the Steklov-Lyapunov systems, the separating variables presented in [8] can also be regarded as new separating variables for (4), (5). 


\section{Conclusive Remarks}

In given paper we gave a justification of the separation of variables and the theta-function solution of the Steklov-Lyapunov systems obtained by F. Kötter [14]. Using the results of [1, 2], we also presented such a solution for an alternative set of variables, which have a simpler form.

On the other hand, there exist several nontrivial integrable generalizations of the systems: the first of them was discovered by V. Rubanovsky [17] and describes a motion of a gyrostat in an ideal fluid under the action of the Archimedes torque, which arises when the barycenter of the gyrostat does not coincide with its volume center. In this generalization the Hamiltonian of the Kirchhoff equations, apart form quadratic terms, contains linear (gyroscopic) terms in $K, p$. Under the change of variables (3), the gyroscopic generalizations of the systems (4), (5) take the form

$$
\dot{z}=z \times(B z-g)-B p \times(B z-g), \quad \dot{p}=p \times(B z-g)
$$

and, respectively,

$$
\dot{z}=p \times(B z-g), \quad \dot{p}=p \times(z-B p),
$$

where $g=\left(g_{1}, g_{2}, g_{3}\right)^{T}$ is a constant vector related to the angular momentum of the rotor inside the gyrostat.

Following [10], these systems admit the following generalizations of Kötter's Lax pair with an elliptic spectral parameter

$$
\begin{gathered}
\dot{L}(s)=[L(s), A(s)], \quad L(s), A(s) \in s o(3), \quad s \in \mathbb{C}, \\
L(s)_{\alpha \beta}=\varepsilon_{\alpha \beta \gamma}\left(\sqrt{s-b_{\gamma}}\left(z_{\gamma}+s p_{\gamma}\right)+g_{\gamma} / \sqrt{s-b_{\gamma}}\right), \\
A(s)_{\alpha \beta}=\varepsilon_{\alpha \beta \gamma} \frac{1}{s} \sqrt{\left(s-b_{\alpha}\right)\left(s-b_{\beta}\right)}\left(b_{\gamma} z_{\gamma}-g_{\gamma}\right), \quad \text { resp. } \quad A(s)_{\alpha \beta}=\varepsilon_{\alpha \beta \gamma} \sqrt{\left(s-b_{\alpha}\right)\left(s-b_{\beta}\right)} p_{\gamma}
\end{gathered}
$$

which provides a sufficient set of constants of motion and makes possible to obtain theta-function solutions. Like in the case of the Steklov-Lyapunov systems, generic invariant manifolds of the Rubanovsky systems are two-dimensional tori, which can be extended to affine parts of Abelian varieties. However, as we plan to show in a forthcoming publication, an explicit integration of the latter systems appears to be more complicated, and the Abelian varieties are not Jacobians of genus 2 hyperelliptic curves, but Prym subvarieties.

The problem of separation of variables for the Rubanovsky systems is still unsolved.

\section{References}

[1] Adler M., van Moerbeke P.: Geodesic flow on so(4) and the intersections of quadrics. Proc.Natl. Acad. Sci. USA. 81, (1984), 4613-4616

[2] Adler M., van Moerbeke P. The complex geometry of the Kowalevski-Painlevé analysis. Invent. Math. 97 (1989), 3-51

[3] Baker H.F. Abels Teorem and the Allied Theory Incluiding the Theory of Theta Functions. Cambridge Univ. Press, Cambridge, 1897

[4] Belokolos E.D., Bobenko A.I., Enol'skii V.Z., Its A.R., and Matveev V.B. Algebro-Geometric Approach to Nonlinear Integrable Equations. Springer Series in Nonlinear Dynamics. SpringerVerlag 1994.

[5] Bobenko A.I. Euler equations in the Lie algebras $e(3)$ and so(4). Isomorphisms of integrable cases.Funkts. Anal. Prolozh. 20, No.1, 64-66 (1986). English transl.:Funct. Anal. Appl. 20 (1986), 53-56

[6] Bolsinov A.V., Fedorov Yu.N. Multidimensional integrable generalizations of the SteklovLiapunov system. Vestnik Mosk. Univ., Ser. I, Math. Mekh. No.6, 53-56 (1992) (Russian) 
[7] Bolsinov A.V., Fedorov Yu.N. Steklov-Lyapunov type systems. Preprint

[8] Bueken P, Vanhaecke P. The moduli problem for integrable systems: the example of a geodesic flow on SO(4). J. London Math. Soc. (2) 62 (2000), no. 2, 357-369

[9] Dubrovin B.A.: Theta-functions and non-linear equations. Usp.Mat. Nauk 36, No.2 (1981), 11-80. English transl.: Russ. Math. Surveys. 36 (1981), 11-92

[10] Fedorov Yu. Integrable systems, Poisson pencils, and hyperelliptic Lax pairs. Regul. Chaotic Dyn. 5 (2000), no. 2, 171-180

[11] Griffiths Ph., Harris J. Principles of Algebraic Geometry. Wiley Interscience, New York 1978

[12] Kolosoff G.V. Sur le mouvement d'un corp solide dans un liquide indéfini. C.R. Acad. Sci. Paris 169 (1919), 685-686

[13] Königsberger L. Zur Transformation der Abelschen Functionen erster Ordnung. J. Reine Agew. Math. 64 (1894), 3-42

[14] Kötter F. Die von Steklow und Liapunow entdeckten integralen Fälle, der Bewegung eines starren Körpers in einer Flüssigkeit. Sitzungsber., König. Preuss. Akad. Wiss., Berlin 6 (1900), $79-87$

[15] Steklov V. On the motion of a rigid body in a fluid. Kharkov, 1903 (Russian)

[16] Lyapunov A.M. New integrable case of the equations of motion of a rigid body in a fluid. Fortschr. Math. 25 (1897), 1501-1504 (Russian)

[17] Rubanovsky V. Integrable cases in the problem of a heavy solid moving in a fluid. Dokl. Akad. Nauk SSSR, 180, (1968), 556-559 (Russian). English transl.: Sov. Phys., Dokl. 13 (1968), 395-397

[18] Tsiganov A. V. On the Steklov-Lyapunov case of the rigid body motion. Regul. Chaotic Dyn. 9 (2004), no. 2, 77-89

[19] Weierstrass K. Mathematische Werke I, vol. 1, 1894 